\title{
Proficiency test scheme for histamine analysis in fishery products in Morocco using the fluorometric method and comparison of results with those obtained with the HPLC reference method
}

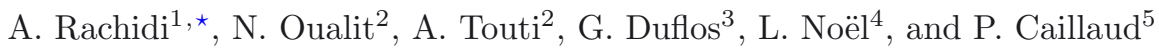 \\ 1 ONSSA, Regional Laboratory of Analysis and Research, Casablanca, Morocco \\ 2 CUMAREX AS, Industrial Area, Tétouan, Morocco \\ 3 ANSES, French Agency for Food, Environmental and Occupational Health \& Safety, Boulogne-sur-Mer Laboratory for Fishery \\ Products, France \\ 4 ANSES Maisons-Alfort Laboratory for Food Safety, Unit for Mineral and Inorganic Contaminants in the Environment, France \\ 5 AFNOR, EURL, Enologues de France, 1 rue des Cèdres, 33290 Blanquefort, France
}

Received: 31 March 2011 / Accepted: 7 November 2011

\begin{abstract}
A Proficiency Test Scheme (PTS) for histamine analysis in fishery products using the Lerke and Bell (1976) fluorometric method was conducted in 26 different Moroccan laboratories. The goal of this PTS was to assess the abilities of a network of seven government laboratories and 19 laboratories performing auto-controls on fish processing units in Morocco. The samples used in the PTS were prepared from canned ground mackerel flesh, to which was added two significant concentrations (levels 1 and 2) of histamine. The homogeneity of the PTS samples was verified by a repeatability test and their stability by an intermediate fidelity test. The Cochran test was used to eradicate anomalous scattering and the Grubbs test to eradicate the anomalous averages observed in the results of the participating laboratories. The average and the usual deviations determined for levels 1 and 2 were used to calculate the $Z$-scores. For level 1 therefore, 23 out of 26 laboratories had satisfactory PTS results, and for level 2, 21 out of 26 laboratories had satisfactory PTS results. An analysis of the PTS samples using the HPLC reference method gave similar results for levels 1 and 2 .
\end{abstract}

Keywords: Proficiency Test Scheme (PTS); histamine; fishery product; fluorometric method; HPLC

\section{Introduction}

The fidelity of an analytical method can affect the reliability of the obtained results [1]. The term fidelity is not without ambiguity. Indeed, the reproducibility of a measurement method depends on the experimental conditions at the time of measurement, and these conditions vary, not only from one laboratory to another, but also from one time to the next in the same laboratory [2]. Consequently, if it is to be thorough, a survey of the fidelity of a measurement method should include determination of inter-laboratory variability, which in turn requires a Proficiency Test Scheme [3].

Health control of fishery products with regard to chemicals essentially relates to the amount of nitrogenous compounds such as total volatile basic nitrogen (TVBN) in fresh and frozen products [4] and the amount of histamine in canned products [5]. Histamine is one of the biogenic amines that are produced by the decarboxylation of amino acids resulting from the action of certain bacteria. In spite of the close relationship between biogenic amine content

` Correspondence: abderrazzak57@gmail.com and sensory change [6], only histamine concentrations in fishery products are regulated for health protection reasons [5]. The objective of our survey was to conduct a Proficiency Test Scheme in order to evaluate the fidelity of histamine analysis in fishery products by the fluorometric method [7]. The official laboratories involved are seeking accreditation according to the ISO/IEC 17025 standard; their participation in the PTS is a requirement of this standard [8]. However, according to regulation (EC) 2073/2005 [5], the reference method for histamine analysis is the HPLC method [9]. The results obtained by the fluorometric method are intended to be compared to those obtained by HPLC.

\section{Materials and methods}

\subsection{Materials}

\subsubsection{Samples for the PTS}

A sufficient quantity of mackerel (about $100 \mathrm{~kg}$ ) was selected from the same fishing batch; the fish was cooked, 


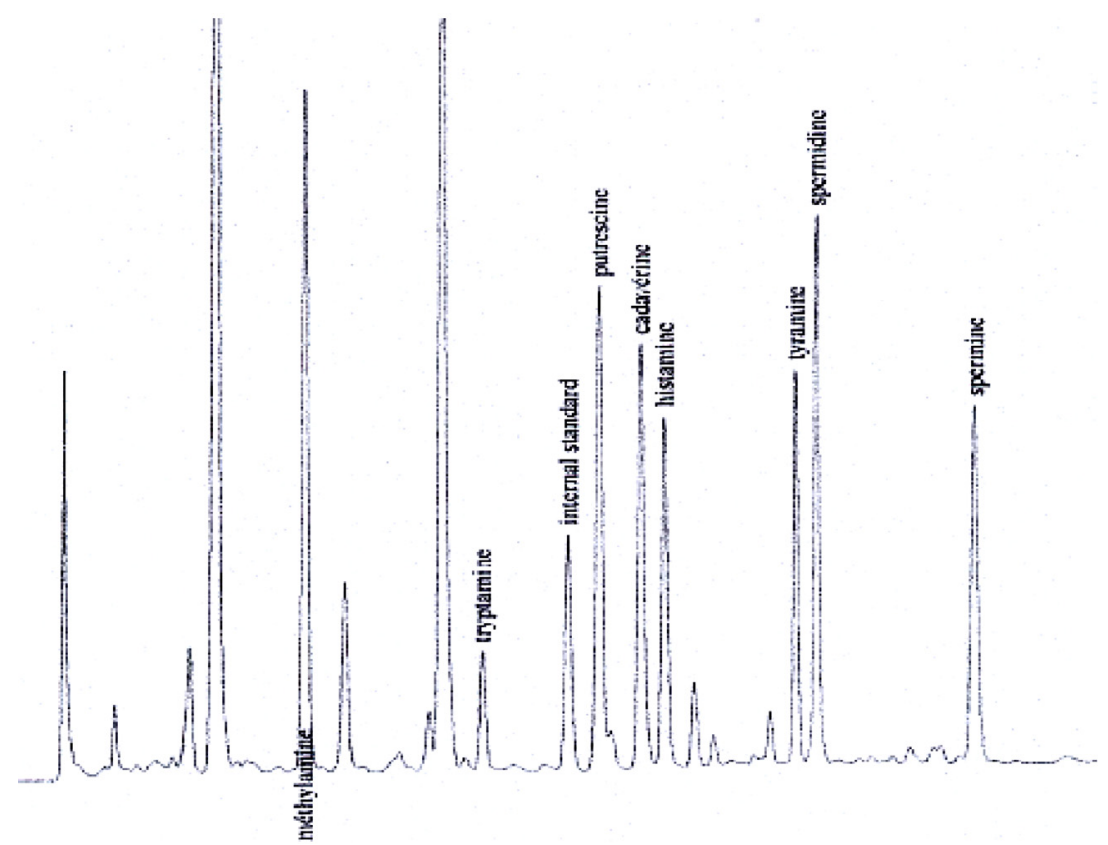

Fig. 1. Reference chromatogram (Duflos et al. [9]).

then cut in slices and ground into a homogeneous paste. The ground mackerel obtained was subdivided into two portions. A histamine solution was added to each one to bring their final histamine concentrations to level 1 and level 2. The two portions of ground fish (level 1 and level 2) were then divided into cans without any accompanying liquid, then they underwent a canning process which respected the parameters of autoclave temperature, pressure and time.

\subsubsection{Reagents}

- Trichloroacetic acid, Scharlau, Spain;

- sodium acetate, Merck, Germany;

- acetic acid, Scharlau, Spain;

- sodium hydroxide, Panreac, Spain;

- amberlite CG50 type I, Fluka, France;

- o-phthalaldehyde. Fluka, Austria;

- dansyl chloride. Sigma, Switzerland;

- histamine dihydrochloride, Fluka, France;

- acetone, HPLC quality, Scharlau, Spain;

- acetonitrile, HPLC quality, Scharlau, Spain;

- toluene, HPLC quality, Merck, Germany;

- perchloric acid 70\%, Merck, Germany;

- 1,3 diaminopropane, Aldrich, Japan;

- sodium carbonate, Scharlau, Spain;

- L-Proline, Sigma, Switzerland.

\subsubsection{Equipment}

- Refrigerated centrifuge, Hermle, Germany;

- homogenizer, Ultra Turrax;

- evaporator under a nitrogen stream, Eyela, Japan;

- chromatography column $(150 \times 9 \mathrm{~mm})$;
- RF-1501 spectrofluorometer, Shimadzu, Japan;

- prominence LC-20A HPLC system, Shimadzu, Japan;

- SIL 20 AC Automatic injector refrigerated to $4{ }^{\circ} \mathrm{C}$, Shimadzu, Japan;

- SPD M 20 A Diode Array Detector, Shimadzu, Japan.

\subsection{Methods}

\subsubsection{Lerke and Bell (1976) fluorometric method [7]}

This method is applied to all fishery products irrespective of their presentation. Histamine is extracted with trichloroacetic acid then fixed on a column filled with an amberlite resin and eluted_by hydrochloric acid. Dosage is done by fluorometry after addition of o-phthalaldehyde. This method was used by all the participating laboratories for the determination of histamine amounts in the levels 1 and 2 of the PTS samples.

\subsubsection{Duflos et al. (1999) HPLC method [9]}

This HPLC method is used for histamine analysis (0 to $250 \mathrm{mg} / \mathrm{kg}$ ) in fresh fish, frozen food and canned food and was performed on Wakosil Column $\mathrm{C}_{18} \mathrm{RS} 5 \mu \mathrm{m}, 100 \AA$ $(25 \mathrm{~cm} \times 4.6 \mathrm{~mm})$. Peaks are identified using the reference chromatogram (Fig. 1).

The chromatographic separation lasts $30 \mathrm{~min}$ as shown in Table 1. This method was used by the LRAR of Casablanca (ONSSA) to compare the PTS results obtained using the fluorometric method [7] and those obtained by the HPLC reference method [5] for the levels 1 and 2 of the PTS samples. 
Table 1. Gradient of elution.

\begin{tabular}{ccc}
\hline Time $(\min )$ & Water & Acetonitrile \\
\hline 0 & $40 \%$ & $60 \%$ \\
6 & $25 \%$ & $75 \%$ \\
8 & $25 \%$ & $75 \%$ \\
13 & $5 \%$ & $95 \%$ \\
20 & $5 \%$ & $95 \%$ \\
20.01 & $40 \%$ & $60 \%$ \\
30 & $40 \%$ & $60 \%$ \\
\hline
\end{tabular}

\subsubsection{Homogeneity test}

For comparison of the histamine distribution in PTS samples, a homogeneity test verified that the histamine concentration was identical for each of the canned fish samples distributed to the participants. Canned fish was tested for Levels 1 and 2. Ten samples were taken randomly from the prepared samples and analysed in duplicate under repeatability conditions according to a validated methodology for homogeneity tests by fluorometric method [7]. The sampling and analytical procedures were in accordance with a recommended procedure [10]. The value of the sampling standard deviation ( $\sigma \mathrm{sam})$ was estimated from the mean squares after one-way analysis of variance (ANOVA), and a statistical test was carried out. This IUPAC Harmonised Protocol for Proficiency Testing [10] requires that $\sigma$ sam should be less than $30 \%$ of the target standard deviation $\sigma p$, that is $\sigma s a m / \sigma p<0.3$. This condition is called 'sufficient homogeneity' in the Harmonised Protocol.

\subsubsection{Stability test}

Ten canned fish samples for each level (1 and 2) were chosen at random for histamine determination repeated five times under the repeatability conditions according to the fluorometric method [7]. Stability was verified for ten weeks during the analysis period of the participating laboratories: from 28 July to 1 October 2010, under intermediate fidelity conditions. The value of the sampling standard deviation ( $\sigma$ sam $)$ was estimated from the mean squares after one-way analysis of variance (ANOVA), and a statistical test was carried out. This IUPAC Harmonised Protocol for Proficiency Testing [10] requires that $\sigma s a m$ should be less than $30 \%$ of the target standard deviation $\sigma p$, that is $\sigma s a m / \sigma p<0.3$. The test of stability permitted to verify that the samples of the PTS (levels 1 and 2) were steady during the whole period of the PTS.

\subsubsection{Statistical analysis of the PTS results}

\subsubsection{Determination of anomalous values}

To perform the statistical analysis of the raw output obtained by the group of participating laboratories, we used AIL 5725 software. This software uses the NF ISO
5725:1994 standard entitled "Accuracy (trueness and precision) of measurement methods and results" [2]. Raw data sent back by participants were analysed using statistical tests according to the ISO Standard 5725 parts 1 and 2. Rejection of partly empty cells was used (non valid data). The statistical analysis allowed the detection of suspect and outlying data between blind duplicates (Cochran's maximum variance test) and between laboratory means (Grubbs, and/or double Grubbs, outlier tests): $p>5 \%$ ( $p$ is the probability of the observed value of the test statistics), i.e., if the Cochran's and/or Grubbs test statistics was less than its $5 \%$ critical value, the item was accepted; if $5 \%>p>1 \%$, i.e., the test statistic lies between $5 \%$ and $1 \%$ critical value, it was a straggler and the test was statistically significant; if $p<1 \%$, i.e., the test statistic was greater than its $1 \%$ critical value, this was a statistical outlier, and the test was highly significant. The outlying data (outliers and extreme values) were eliminated, and the precision data, mean, standard deviation, coefficient of variation of repeatability $\left(C V_{r}\right)$ and reproducibility $\left(C V_{R}\right)$ were calculated. The assigned values (AV) obtained from the PTS were adopted as the best available estimate of the true values for the analysts in the test materials and used as external target values for PTS.

\subsubsection{Assessment of the participating laboratories after calculation of the Z-scoress}

The ISO/IEC 17043 (2010) Conformity assessment General requirements for proficiency testing Annex B.3.1.3. [11], requires the average obtained by each of the participants to be converted into a " $Z$-scores" [3]. In this document, the use of this system is based on the calculation of intervals. A participant's " $m$ " result is converted into a $Z$-scores, according to the formula:

$$
Z=\frac{(m-M)}{\sigma}
$$

where $M$ is the general average or the conventionally true value of the content in the analyte and $\sigma$ is the "ideal" standard deviation of inter-laboratory reproducibility that reflects the highest variation accepted among laboratories.

\section{Results and discussion}

\subsection{Results of the homogeneity test}

For the histamine content, determined by the laboratory in the repeatability conditions (5 repetitions on ten different analysis samples), in the level 1 PTS samples, the general average was $40.2 \mathrm{mg} / \mathrm{kg}$, the total variance was $1.31 \mathrm{mg} / \mathrm{kg}$ and the total coefficient of variation was $3.3 \%$. For level 2, the general average was $69.2 \mathrm{mg} / \mathrm{kg}$, the total variance was $0.922 \mathrm{mg} / \mathrm{kg}$ and the total coefficient of variation was $1.3 \%$.

The Cochran test showed that, for the histamine results obtained on the levels 1 and 2 of the PTS samples, 


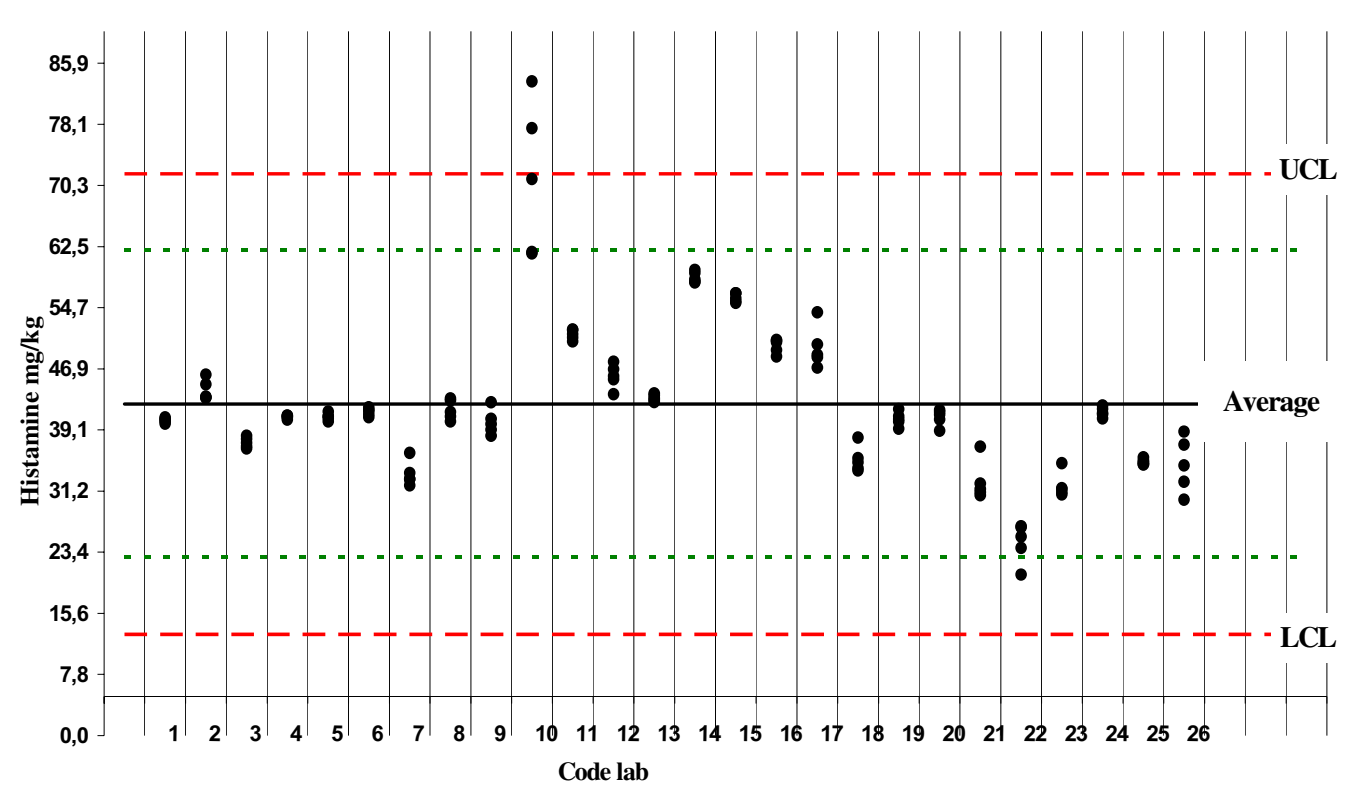

UCL: Upper Control Limit

LCL: Lower Control Limit

Fig. 2. (Color online) Histamine $(\mathrm{mg} / \mathrm{kg})$, in the level 1 matrix obtained by the 26 participating laboratories.

the biggest scatterings (coefficient of variation) were acceptable. Similarly, the Grubbs test showed that, for the histamine results obtained on the levels 1 and 2 of the PTS samples, the smallest and biggest averages were acceptable. The homogeneity of the levels 1 and 2 of the PTS samples was then verified.

\subsection{Results of the stability test}

For the histamine content, determined by the laboratory in the intermediate fidelity conditions ( 5 repetitions on ten different analysis samples performed every week), in the level 1 of the PTS samples, the general average was $40.3 \mathrm{mg} / \mathrm{kg}$, the total variance was $0.862 \mathrm{mg} / \mathrm{kg}$ and the total coefficient of variation was $2.1 \%$. For level 2, the general average was $69.2 \mathrm{mg} / \mathrm{kg}$, the total variance was $0.855 \mathrm{mg} / \mathrm{kg}$ and the total coefficient of variation was $1.2 \%$. The Cochran test showed that, for the results of histamine obtained on the levels 1 and 2 of the PTS samples, the biggest scatterings (coefficient of variation) were acceptable. Similarly, the Grubbs test showed that, for the histamine results obtained on the levels 1 and 2 of the PTS samples, the smallest and biggest averages were acceptable. The stability of the levels 1 and 2 of the PTS samples was then verified.

\subsection{Results of the proficiency test scheme for the level 1 sample}

3.3.1 Unprocessed and corrected (parametrical statistics) data for the level 1 of the PTS sample

Table 2 and Figure 2 present the unprocessed and corrected data for histamine expressed in $\mathrm{mg} / \mathrm{kg}$, in the level 1 matrix obtained by the 26 participating laboratories. The variance of laboratory number 17 was significant at the $5 \%$ level according to the Cochran test, but these data were not eradicated. The variance in laboratories 10 and 26 were significant at the $1 \%$ level, the data from these labs were therefore removed from the full data set. The averages of laboratories 10 and 14 were detected as abnormal by the Grubbs test, the data from these laboratories were therefore removed from the full data set. The individual performance of laboratories 10, 14 and 26 were not therefore taken into account for this test in the calculations of the overall average and the standard deviation.

\subsubsection{Parametrical statistical balance for the level 1 of the PTS sample}

The statistical balance of the histamine dosage in the level 1 matrix is indicated in Table 3 . The general average of the histamine concentration of the level 1 matrix was $40.7 \mathrm{mg} / \mathrm{kg}$. This is presumably the conventionally true value of the histamine content in the level 1 sample. The coefficients of variation of fidelity for repeatability $(3.3 \%)$ and reproducibility (17.4\%) are acceptable.

\subsubsection{Results of the $Z$-scores for the level 1 PTS sample}

Table 4 and Figure 3 show the $Z$-score results for the determination of histamine in the level 1 PTS sample after elimination of the abnormal variance data. The data from laboratory number 10 were judged as abnormal and were not taken into consideration. The data from laboratories 22,15 and 14 are questionable according to the application of $Z$-scores $(2.0<|Z| \leq 3.0)$. For the other laboratories, the data was satisfactory $(|Z| \leqslant 2.0)$. 
Table 2. Histamine $(\mathrm{mg} / \mathrm{kg})$, in the level 1 matrix obtained by the 26 participating laboratories.

\begin{tabular}{|c|c|c|c|c|c|c|c|}
\hline Code lab & Test 1 & Test 2 & Test 3 & Test 4 & Test 5 & Averages & $\begin{array}{l}\text { Standard } \\
\text { deviation }\end{array}$ \\
\hline 1 & 39.9 & 40.3 & 40.5 & 40.6 & 40.1 & 40.3 & 0.30 \\
\hline 2 & 43.3 & 46.1 & 43.2 & 44.9 & 43.2 & 44.1 & 1.32 \\
\hline 3 & 37.4 & 38.3 & 37.0 & 36.7 & 37.9 & 37.4 & 0.66 \\
\hline 4 & 40.4 & 40.6 & 40.9 & 40.9 & 40.7 & 40.7 & 0.22 \\
\hline 5 & 40.1 & 40.8 & 40.5 & 41.4 & 40.8 & 40.7 & 0.47 \\
\hline 6 & 40.7 & 41.6 & 41.5 & 42.0 & 41.0 & 41.3 & 0.51 \\
\hline 7 & 32.8 & 33.5 & 36.1 & 32.8 & 32.0 & 33.4 & 1.61 \\
\hline 8 & 43.1 & 41.3 & 40.8 & 40.1 & 42.8 & 41.6 & 1.28 \\
\hline 9 & 38.3 & 40.5 & 42.6 & 39.1 & 39.8 & 40.0 & 1.64 \\
\hline 10 & 61.8 & 71.1 & 83.6 & 77.6 & 61.6 & 71.1 & 9.69 \\
\hline 11 & 51.9 & 50.9 & 50.3 & 51.3 & 51.8 & 51.2 & 0.65 \\
\hline 12 & 47.8 & 45.9 & 45.5 & 43.6 & 46.8 & 45.9 & 1.55 \\
\hline 13 & 43.7 & 42.9 & 43.1 & 43.5 & 42.6 & 43.2 & 0.43 \\
\hline 14 & 59.1 & 58.3 & 58.0 & 57.9 & 59.5 & 58.6 & 0.71 \\
\hline 15 & 55.5 & 55.3 & 55.9 & 56.5 & 56.5 & 55.9 & 0.55 \\
\hline 16 & 48.4 & 50.3 & 50.5 & 50.5 & 49.3 & 49.8 & 0.92 \\
\hline 17 & 50.0 & 54.1 & 48.3 & 47.0 & 48.7 & 49.6 & 2.71 \\
\hline 18 & 33.9 & 34.2 & 35.4 & 38.1 & 34.9 & 35.3 & 1.67 \\
\hline 19 & 40.1 & 40.8 & 39.2 & 40.4 & 41.7 & 40.4 & 0.92 \\
\hline 20 & 41.3 & 41.6 & 41.0 & 38.9 & 40.4 & 40.7 & 1.06 \\
\hline 21 & 36.9 & 31.1 & 32.2 & 30.7 & 31.5 & 32.5 & 2.53 \\
\hline 22 & 20.6 & 26.6 & 26.7 & 24.0 & 25.4 & 24.7 & 2.55 \\
\hline 23 & 31.5 & 31.1 & 34.8 & 31.6 & 30.8 & 32.0 & 1.62 \\
\hline 24 & 40.5 & 41.2 & 41.0 & 41.8 & 42.2 & 41.3 & 0.64 \\
\hline 25 & 35.5 & 34.7 & 35.1 & 34.6 & 34.9 & 35.0 & 0.37 \\
\hline 26 & 30.1 & 34.5 & 37.2 & 32.4 & 38.8 & 34.6 & 3.51 \\
\hline
\end{tabular}

Table 3. Statistical balance of the laboratory network for the histamine amount $(\mathrm{mg} / \mathrm{kg})$, in the level 1 PTS sample.

\begin{tabular}{l|c}
\hline Parametric average: & $x=40.7$ \\
Repeatability standard deviation: & $s_{r}=1.4$ \\
Repeatability coefficient of variation: & $C V_{r}=3.3 \%$ \\
Inter laboratories standard deviation: & $s_{L}=7.0$ \\
Inter laboratories coefficient of variation: & $C V_{L}=17.1 \%$ \\
Reproductibility standard deviation: & $s_{R}=7.1$ \\
Reproductibility coefficient of variation: & $C V_{R}=17.4 \%$ \\
Number of laboratories after withdrawal of the abnormal: & $n=23.0$ \\
\hline
\end{tabular}

\subsection{Results of the proficiency test scheme for the level 2 sample}

\subsubsection{Unprocessed and corrected (parametrical statistics) data for the level 2 PTS sample}

Table 5 and Figure 4 present the unprocessed and corrected data of the histamine expressed in $\mathrm{mg} / \mathrm{kg}$, in the level 2 matrix (sample: PTS 2010 - level 2) obtained by the 26 participating laboratories. The variances of the laboratories 22, 10 and 16 were detected as abnormal by the Cochran test to $1 \%$, the data from these labs were therefore eradicated from the full set. The averages of laboratories 10, 16, 21 and 23 were detected as abnormal by the Grubbs test; the data from these laboratories were therefore eradicated from the full set. The individual 


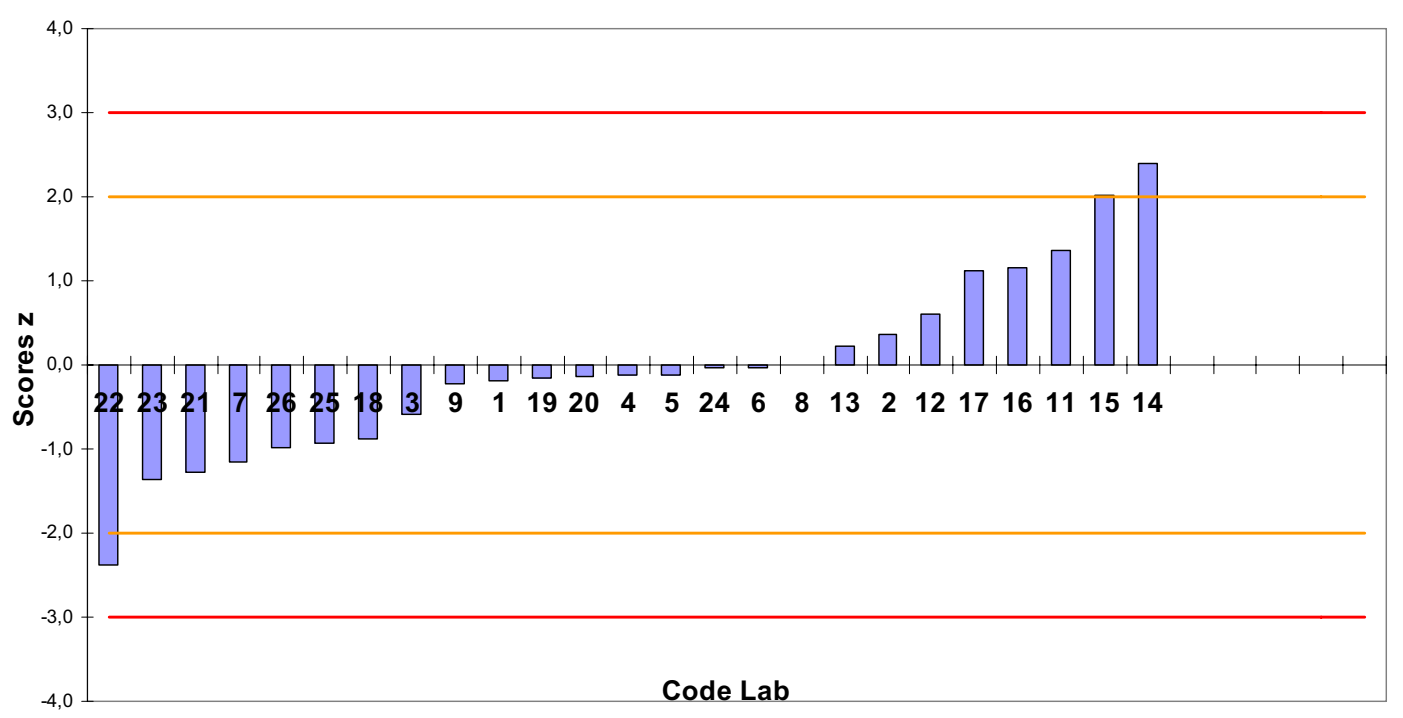

Fig. 3. (Color online) $Z$-score obtained by the laboratories for the histamine amount in the level 1 PTS sample.

Table 4. $Z$-score obtained by the laboratories for the histamine amount in the level 1 PTS sample.

\begin{tabular}{|c|c|c|}
\hline Code lab & Average & $Z$-scores \\
\hline 1 & 40.3 & -0.18 \\
\hline 2 & 44.1 & 0.36 \\
\hline 3 & 37.4 & -0.58 \\
\hline 4 & 40.7 & -0.12 \\
\hline 5 & 40.7 & -0.12 \\
\hline 6 & 41.3 & -0.03 \\
\hline 7 & 33.4 & -1.15 \\
\hline 8 & 41.6 & 0.01 \\
\hline 9 & 40.0 & -0.22 \\
\hline 11 & 51.2 & 1.36 \\
\hline 12 & 45.9 & 0.61 \\
\hline 13 & 43.2 & 0.22 \\
\hline 14 & 58.6 & 2.39 \\
\hline 15 & 55.9 & 2.02 \\
\hline 16 & 49.8 & 1.16 \\
\hline 17 & 49.6 & 1.13 \\
\hline 18 & 35.3 & -0.89 \\
\hline 19 & 40.4 & -0.16 \\
\hline 20 & 40.6 & -0.13 \\
\hline 21 & 32.5 & -1.28 \\
\hline 22 & 24.7 & -2.38 \\
\hline 23 & 32.0 & -1.35 \\
\hline 24 & 41.3 & -0.04 \\
\hline 25 & 35.0 & -0.93 \\
\hline 26 & 34.6 & -0.98 \\
\hline
\end{tabular}

performance of laboratories 10, 16, 21, 22 and 23 were not taken into account for this test in the calculations of the average and the general standard deviation.

\subsubsection{Parametrical statistical balance for the level 2 PTS sample}

The statistical balance of the histamine dosage in the level 2 matrix is indicated in Figure 6. The general average of the histamine concentration of the level 2 PTS sample was $73.3 \mathrm{mg} / \mathrm{kg}$. This is presumably the conventionally true value of the histamine content in the level 2 sample. The coefficients of variation of fidelity for repeatability $(2.3 \%)$ and reproducibility $(18.0 \%)$ are acceptable.

\subsubsection{Results of the $Z$-scores \\ for the level 2 of the PTS sample}

Table 7 and Figure 5 show the $Z$-scores results for the determination of histamine in the level 2 of the PTS sample after eradication of the abnormal variance data. The results from laboratories 10 and 22 were judged as incorrect and have not been considered $(|Z| \geq 3.0)$. The data from laboratories $12,14,15$ and 16 are questionable according to this test $(2.0<|Z|<3.0)$. For the other laboratories, the data was satisfactory $(|Z| \leq 2.0)$.

\subsection{Results of the dosage of the level 1 and level 2 of the PTS sample by the HPLC reference method}

The results of the dosage of the level 1 and level 2 PTS sample are presented in Table 8 and illustrated by Figure 6 . The histamine concentration measured by HPLC in the level 1 sample was $40.9 \mathrm{mg} / \mathrm{kg}$ and that of the level 2 sample was $73.5 \mathrm{mg} / \mathrm{kg}$. 


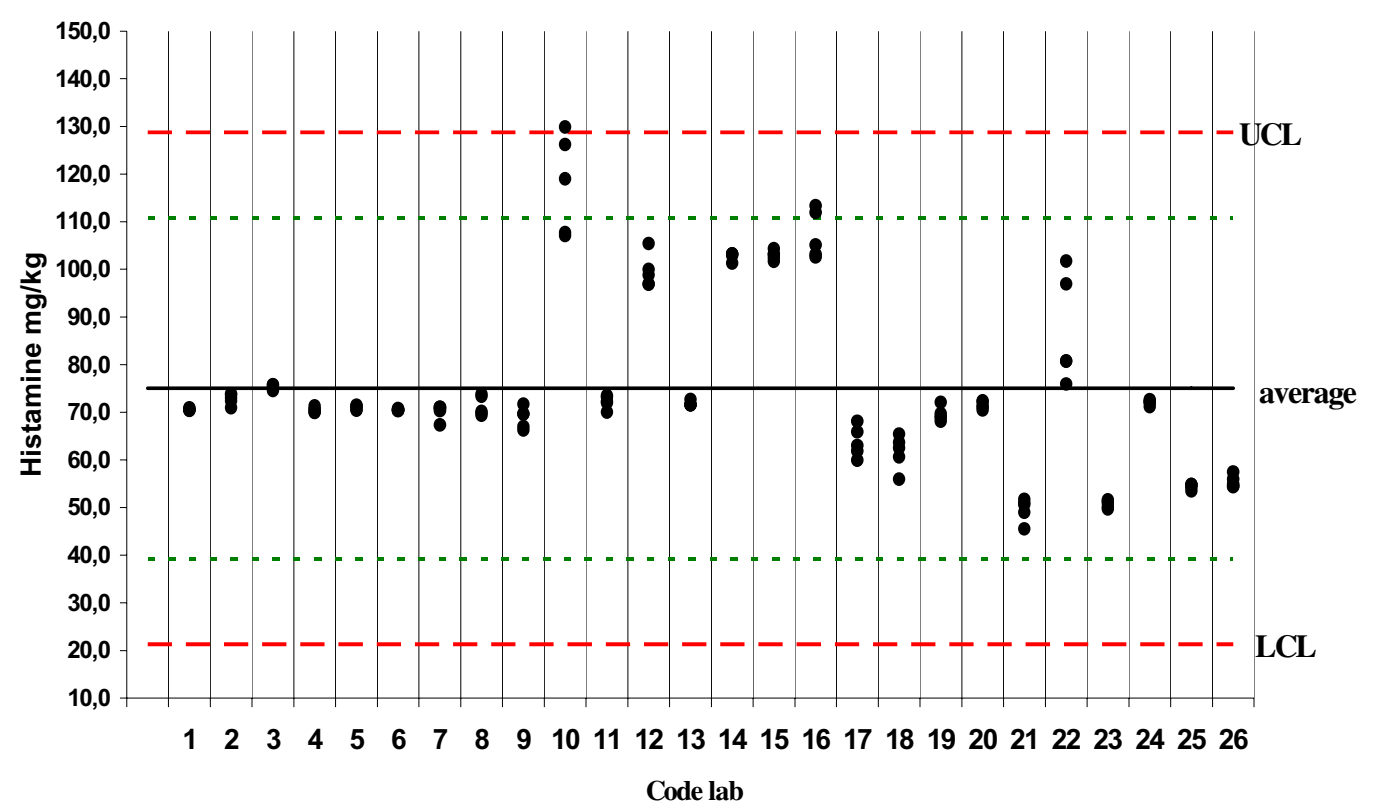

UCL: Upper Control Limit

LCL: Lower Control Limit

Fig. 4. (Color online) Histamine $(\mathrm{mg} / \mathrm{kg})$, in the level 2 matrix obtained by the 26 participating laboratories.

Table 5. Histamine $(\mathrm{mg} / \mathrm{kg})$, in the level 2 matrix obtained by the 26 participating laboratories.

\begin{tabular}{cccccccc}
\hline Code lab & Test 1 & Test 2 & Test 3 & Test 4 & Test 5 & Averages & $\begin{array}{c}\text { Standard } \\
\text { deviation }\end{array}$ \\
\hline 1 & & & & & & & 0.23 \\
2 & 70.4 & 70.5 & 70.5 & 70.7 & 70.9 & 70.6 & 1.19 \\
3 & 75.2 & 73.8 & 72.4 & 70.9 & 73.0 & 72.8 & 0.51 \\
4 & 70.5 & 71.3 & 70.6 & 70.0 & 70.0 & 70.5 & 0.57 \\
5 & 71.2 & 71.5 & 70.5 & 70.4 & 71.2 & 71.0 & 0.46 \\
6 & 70.5 & 70.8 & 70.3 & 70.4 & 70.4 & 70.5 & 0.18 \\
7 & 70.8 & 71.1 & 71.0 & 67.3 & 70.3 & 70.1 & 1.57 \\
8 & 73.8 & 69.4 & 70.2 & 69.8 & 73.3 & 71.3 & 2.10 \\
9 & 66.3 & 69.6 & 67.0 & 71.7 & 69.7 & 68.9 & 2.19 \\
10 & 107.7 & 119.0 & 129.9 & 107.1 & 126.2 & 118.0 & 10.4 \\
11 & 72.3 & 73.5 & 73.1 & 72.0 & 70.0 & 72.2 & 1.35 \\
12 & 105.4 & 96.9 & 96.9 & 98.8 & 100.0 & 99.6 & 3.51 \\
13 & 71.6 & 71.6 & 71.5 & 72.7 & 71.6 & 71.8 & 0.51 \\
14 & 102.9 & 101.3 & 103.2 & 103.2 & 103.2 & 102.8 & 0.83 \\
15 & 104.3 & 101.7 & 103.2 & 103.1 & 102.4 & 102.9 & 0.97 \\
16 & 103.2 & 102.6 & 113.3 & 105.1 & 111.9 & 107.2 & 5.05 \\
17 & 65.9 & 61.9 & 59.9 & 63.0 & 68.1 & 63.8 & 3.24 \\
18 & 60.6 & 62.5 & 55.9 & 63.7 & 65.4 & 61.6 & 3.63 \\
19 & 69.1 & 69.7 & 72.1 & 68.8 & 68.1 & 69.6 & 1.53 \\
20 & 72.4 & 71.0 & 70.5 & 71.4 & 72.3 & 71.5 & 0.81 \\
21 & 50.6 & 51.0 & 45.5 & 51.7 & 49.0 & 49.6 & 2.48 \\
22 & 75.9 & 80.7 & 80.8 & 101.7 & 97.0 & 87.2 & 11.4 \\
23 & 51.3 & 49.7 & 50.9 & 51.6 & 50.1 & 50.7 & 0.80 \\
24 & 71.2 & 72.0 & 72.6 & 72.3 & 72.0 & 72.0 & 0.55 \\
25 & 54.3 & 53.5 & 54.8 & 54.6 & 54.9 & 54.4 & 0.58 \\
26 & 55.0 & 54.5 & 55.9 & 54.4 & 57.5 & 55.4 & 1.28 \\
\hline & & & & & & & \\
\hline
\end{tabular}




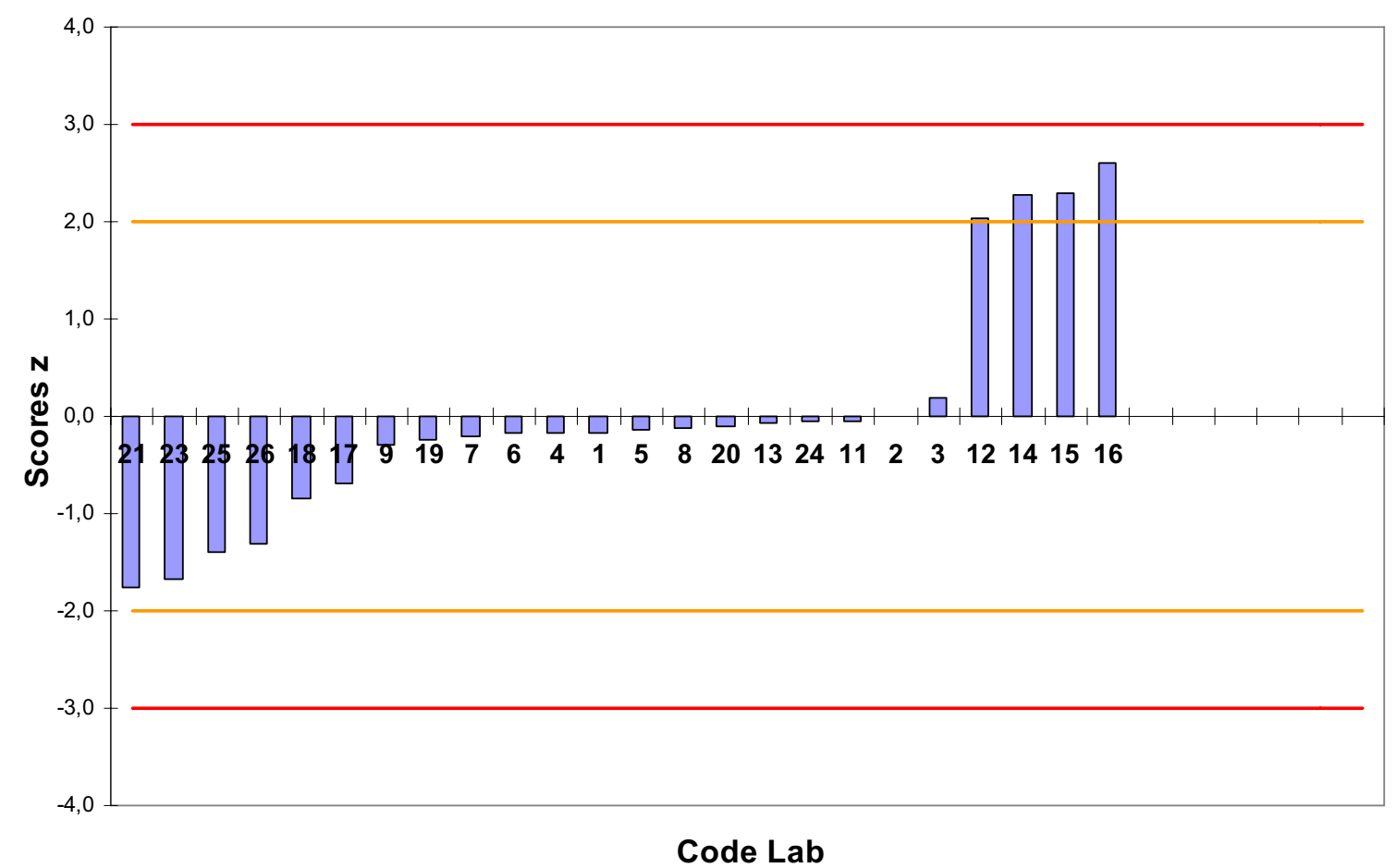

Fig. 5. (Color online) $Z$-scores obtained by the laboratories for the histamine amount in the level 2 PTS sample.

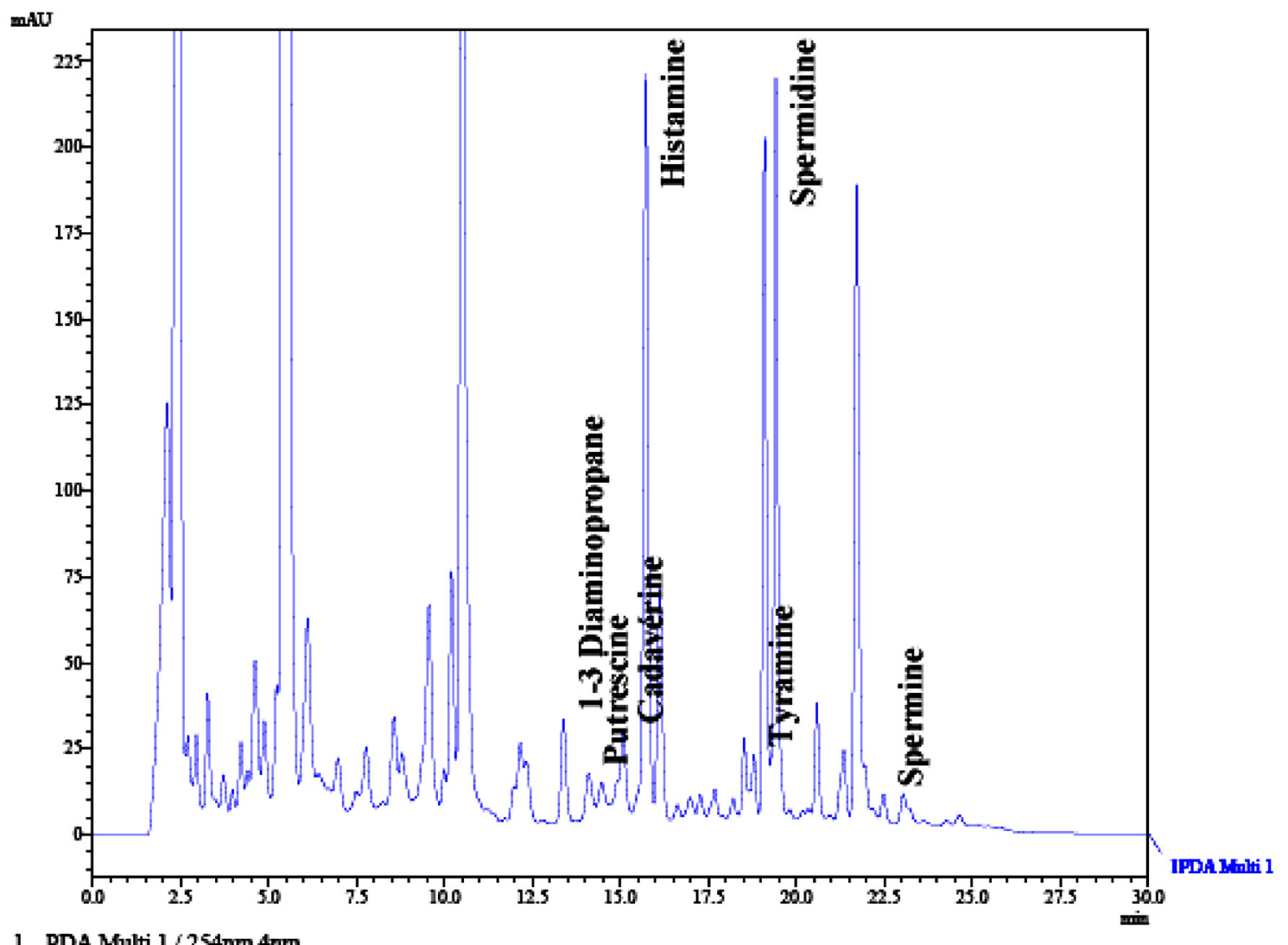

Fig. 6. (Color online) HPLC chromatogram of histamine amount, in the level 1 PTS sample. 
Table 6. Statistical balance of the laboratory network for the histamine amount $(\mathrm{mg} / \mathrm{kg})$, in the level 2 PTS sample.

\begin{tabular}{l|c}
\hline Parametric average: & $x=73.3$ \\
Repeatability standard deviation: & $s_{r}=1.7$ \\
Repeatability coefficient of variation: & $C V_{r}=2.3 \%$ \\
Inter laboratories standard deviation: & $s_{L}=13.1$ \\
Inter laboratories coefficient of variation: & $C V_{L}=17.8 \%$ \\
Reproductibility standard deviation: & $s_{R}=13.2$ \\
Reproductibility coefficient of variation: & $C V_{R}=18.0 \%$ \\
Number of laboratories after withdrawal of the abnormal: & $n=21.0$ \\
\hline
\end{tabular}

Table 7. $Z$-score obtained by the laboratories for the histamine amount in the level 1 PTS sample.

\begin{tabular}{ccc}
\hline Code lab & Average & $Z$-score \\
\hline 1 & 70.6 & -0.17 \\
2 & 72.8 & 0.00 \\
3 & 75.2 & 0.19 \\
4 & 70.5 & -0.18 \\
5 & 71.0 & -0.14 \\
6 & 70.5 & -0.18 \\
7 & 70.1 & -0.20 \\
8 & 71.3 & -0.11 \\
9 & 68.9 & -0.30 \\
11 & 72.2 & -0.05 \\
12 & 99.6 & 2.03 \\
13 & 71.8 & -0.08 \\
14 & 102.8 & 2.27 \\
15 & 102.9 & 2.29 \\
16 & 107.2 & 2.61 \\
17 & 63.8 & -0.69 \\
18 & 61.6 & -0.85 \\
19 & 69.6 & -0.25 \\
20 & 71.5 & -0.10 \\
21 & 49.6 & -1.76 \\
23 & 50.7 & -1.67 \\
24 & 72.0 & -0.06 \\
25 & 54.4 & -1.40 \\
26 & 55.4 & -1.32 \\
\hline & & \\
& &
\end{tabular}

\section{Discussion}

This PTS for histamine analysis in fishery products using the Lerke and Bell fluorometric method [7], made it possible to determine a laboratory's performance while classifying it in relation to the other participants in order to ensure the quality of its results to its customers [3], and to guarantee compliance with the ISO/IEC 17025 reference standard [8]. At the end of this PTS, 21 out of the 26 laboratories obtained satisfactory results regardless of the histamine concentration in the PTS sample (levels 1 and 2). However, the number of laboratories not satisfying the PTS for the level 2 sample was greater than that observed for level 1 . It would be advisable for the
Table 8. Results of the histamine amount by the HPLC method in the levels 1 and 2 PTS samples.

\begin{tabular}{cc|cc}
\hline \multicolumn{2}{c|}{$\begin{array}{c}\text { PTS sample } \\
\text { to level 1 }\end{array}$} & \multicolumn{2}{c}{$\begin{array}{c}\text { PTS sample } \\
\text { to level 2 }\end{array}$} \\
\hline Repetitions & $\begin{array}{c}\text { Histamine } \\
\mathrm{mg} / \mathrm{kg}\end{array}$ & $\begin{array}{c}\text { Repetitions } \\
\text { Histamine } \\
\mathrm{mg} / \mathrm{kg}\end{array}$ \\
\hline 1 & 44.8 & 1 & 76.1 \\
2 & 40.2 & 2 & 78.1 \\
3 & 39.2 & 3 & 82.1 \\
4 & 42.4 & 4 & 80.2 \\
5 & 39.7 & 5 & 66.2 \\
6 & 39.4 & 6 & 67.4 \\
7 & 43.2 & 7 & 70.9 \\
8 & 38.7 & 8 & 67.3 \\
\hline Average & 40.9 & Average & 73.5 \\
\hline Standard & 2.09 & Standard & 5.96 \\
deviation & & deviation \\
\hline
\end{tabular}

next PTS to increase the number of levels in order to cover the entire domain of application (0 to $200 \mathrm{mg} / \mathrm{kg}$ of histamine). Moreover, it seems obvious that in examining the deviations between the values obtained by a laboratory and the value assigned for the comparison, interesting information can be obtained on the bias or accuracy of the laboratory. This information is even more applicable than the comparisons which are organised regularly throughout the year. The unsatisfactory results are a warning to the laboratories concerned that they need to identify the reasons for this bias and apply corrective measures.

The coefficients of inter-laboratory variations of reproducibility for level $1\left(C V_{R} 17.4 \%\right)$ and level $2\left(C V_{R} 18.0 \%\right)$ are comparable. The PTS also made it possible to evaluate the measurement uncertainties of a trial method while using the application of the method and the data obtained at the time of the PTS $[3,12]$, as the inter-laboratory coefficients of variations of reproducibility.

The average of the results obtained by the HPLC reference method [9] was comparable to that of the fluorometric method [7] obtained by all the laboratories for levels 1 and 2 and taking into consideration the inter-laboratory coefficients of reproducibility. It would be appropriate to apply statistical analysis to the relationship between the results of the two methods at the time of the next PTS. 


\section{Conclusions}

The PTS for analysing the amount of histamine in fishery products by the fluorometric method [7] identified five laboratories out of 26 that did not satisfy the PTS, and that are invited to identify the bias in their results in order to apply corrective measures. At the time of the next PTS, additional concentrations will be proposed in order to cover the test method's domain of application because the choice of matrix/concentration pair is an important parameter for conducting the PTS. The increase in frequency of this PTS throughout the year is an important factor in the interpretation of the results. The PTS also yielded interesting data for calculating the uncertainty of the test method. Analysing the PTS samples at different concentration levels by the HPLC reference method gave some results comparable to those obtained by the fluorometric method. However, a more elaborate statistical survey for comparing results is necessary to confirm this observation.

\section{References}

1. AFNOR, NF ISO 5725-1, Application de la statistique, Exactitude (justesse et fidélité) des résultats et méthodes de mesure, Partie 1 : Principes généraux et définitions (AFNOR Ed., Paris, 1994), p. 24

2. AFNOR, NF ISO 5725-2, Application de la statistique, Exactitude (justesse et fidélité) des résultats et méthodes de mesure, Partie 2: Méthode de base pour la détermination de la répétabilité et de la reproductibilité d'une méthode de mesure normalisée (AFNOR Ed., Paris, 1994), p. 54
3. NF ISO 13528, Méthodes statistiques utilisées dans les essais d'aptitude par comparaison interlaboratoires (2005)

4. Commission Regulation (EC) No. 2074/2005 of 5 December 2005 laying down implementing measures for certain products under Regulation (EC) No. 853/2004 of the European Parliament and of the Council

5. Commission Regulation (EC) No. 2073/2005 of 15 November 2005 on microbiological criteria for foodstuffs

6. A. Rachidi, M. Alvinerie, A. Dalal, Évaluation de l'état de fraîcheur des produits de la pêche I, Intérêt et limites de l'évaluation sensorielle et chimique du poisson frais, Revue de Médecine Vétérinaire 146, 327-332 (1995)

7. P.A. Lerke, L.D. Bell, A rapid fluorometric method for the determination of histamine in canned tuna, J. Food Sci. 41, 1282-1284 (1976)

8. ISO/IEC 17025, General requirements for the competence of testing and calibration laboratories, 2nd. edn., 2005-0515, Corrected version 2005-11-01

9. G. Duflos, C. Dervin, P. Malle, Relevance of matrix effect in determination of biogenic amines in plaice (Pleuronectes platessa) and whiting (Merlangus merlangus), Journal of AOAC International 82, 1097-1101 (1999)

10. M. Thompson, R. Wood, The international harmonized protocol for the proficiency testing of (chemical) analytical laboratories - A recommended procedure for testing material for sufficient homogeneity (Annex 1), Pure Appl. Chem. 65, 2123-2144 (1993)

11. ISO/IEC 17043, Conformity assessment - General requirements for proficiency testing (2010)

12. AFNOR, FD ISO/TS 21748, Lignes directrices relatives à l'utilisation d'estimations de la répétabilité, de la reproductibilité et de la justesse dans l'évaluation de l'incertitude de mesure (AFNOR Ed., Paris, 2004), p. 32 